\title{
EVALUACIÓN DE DE UNA BEBIDA LÁCTEA INSTANTÁNEA A BASE DE HARINA DE ARRACACHA (Arracacia xanthorrhiza) CON LA ADICIÓN DE ÁCIDO FÓLICO
}

\author{
EVALUATION OF A MILKY INSTANT DRINK \\ WITH ARRACACHA FLOUR (Arracacia xanthorrhiza) \\ AND ADDITION OF FOLIC ACID
}

\author{
Auris García M., Emperatriz Pacheco-Delahaye \\ Laboratorio de Bioquímica de Alimentos del Instituto de Química y Tecnología, \\ Facultad de Agronomía, Universidad Central de Venezuela, Maracay, Estado Aragua, Venezuela.
}

\begin{abstract}
To diversify the use of extruded arracacha flour, it was proposed the preparation of a drink with the addition of folic acid. The methodology applied allowed to determine the chemical composition, physical-chemical characteristics, functional in vitro starch digestibility and shelf stability. The formulation based on a meal arracacha $30,30 \%, 42,32 \%, 27,22 \%$ and $0,16 \%$ of vanilla was the highest preference. It is characterized by intermediate rate of digestion and to the development of a viscous suspension meringue type, no lumps, no phase separation, by high water absorption, swelling power and solubility. It was estimated that $200 \mathrm{~g}$ of the product provides 400 to $340 \mathrm{mg}$ of folic acid and a good supply of protein, minerals and dietary fiber. This mixture had stability for three months of storage. In conclusion this flour can be used as an alternative source of starch to make milk drinks. Key words: Arracacia xanthorriza; arracacha; milk drinks; in vitro starch digestibility; instant mixes.
\end{abstract}

Este trabajo fue recibido el 1 de Abril de 2009, aceptado con modificaciones el 15 de Marzo de 2010 y aceptado para ser publicado el 15 de Noviembre de 2010.

\section{INTRODUCCIÓN}

Las bebidas instantáneas surgieron como una alternativa a las necesidades de una población, que requiere del uso y consumo de alimentos de rápida preparación. Se han considerado vehículos apropiados para hacerles llegar una variedad de nutrientes que aporten beneficios a la salud en la medida que se incluyan con frecuencia en la dieta diaria. Los investigadores mantienen una continua búsqueda de nuevas fuentes alternativas de materias primas para elaborar este tipo de producto, estableciendo el desarrollo de mezclas óptimas de ingredientes, que conlleven a características organolépticas, físicas, químicas y funcionales deseables, y se adapten a los gustos de los consumidores $(1,2)$.

Las bebidas lácteas instantáneas son mezclas deshidratadas, en polvo o granuladas, elaboradas con una relación mínima de $30 \%$ de leche en el producto final, Puede contener agregados de otros ingredientes de uso alimentarios permitidos, entre ellos, cereales, azúcares, saborizantes de frutas, chocolate, vainilla, edulcorantes, frutas o vegetales deshidratadas (3). Sin embargo, la norma Ibernorca (4) define a estas bebidas como una mezcla que al reconstituirla por simple dilución en agua potable, son capaces de solubilizarse e hincharse en agua fría o templada, aumentando su volumen y conformando una solución viscosa de manera inmediata y sin presencia de grumos.

Esta misma norma recomienda para la formulación de las mezclas, utilizar harinas o almidones gelatinizados o pregelatinizados en una relación de sólidos que proporcione las propiedades gelificantes requeridas dependiendo del tipo de bebida (chicha, merengada u otra). Además sugieren que presenten un contenido de humedad máximo de 5\%, color, sabor, olor y consistencia hasta el final del periodo de almacenamiento típico del producto reconstituido y un contaje máximo de 
mohos de 1x102 ucf/g y acidoúricos de 1x103 ucf/g.

Pacheco (5) propuso que estas bebidas sean preferiblemente elaboradas a partir de mezclas de polvos, debido a la alta demanda que tienen en el mercado popular, no sólo por las ventajas que presenta de menor costo durante la manipulación, largo tiempo de vida útil, sino también por ser de fácil preparación. Arcila y Mendoza (6) coinciden con estas premisas, e indican que las bebidas de mayor gusto, son las lácteas del tipo merengada y chicha, las cuales se caracterizan por tener efecto en la reducción de la saciedad y la sensación de hambre en un corto tiempo, después de ser consumidas, resultando de alta satisfacción por los consumidores.

Pacheco et al. (7) sugieren utilizar en su preparación las harinas pre-gelatinizadas y gelatinizadas de raíces y tubérculos por considerar que son una fuente alternativa importante, que puede permitir la sustitución total o parcial de la dependencia de la harina de trigo, y que pueden hacer llegar a la población una gran variedad de nutrientes. Al respecto, explican que estos tipos de harinas, aunque tienen un bajo contenido de grasas, poseen ciertas cantidades proteínas, minerales e incluso de fibra dietética, las cuales pueden elevar su valor nutricional e incluso promover efectos fisiológicos positivos, en función a la disponibilidad de proteínas, minerales y fibra dietaría. En este último caso disminuyen el tiempo de tránsito intestinal y el colesterol, entre otros beneficios.

Dada la importancia de estos materiales amiláceos, se ha propuesto el uso de las harinas extrudidas para la preparación de las mezclas en polvo de bebidas instantáneas, ya que el proceso de extrusión conlleva a la pre-gelatinización de los gránulos de almidón, provocando la pérdida del orden molecular y la completa degradación de los polímeros con la formación de fragmentos altamente solubles, que al incorporarse a este tipo de formulaciones promueve el incremento en la viscosidad por el alto poder de hinchamiento en frío que estos desarrollan, manteniendo su estabilidad como consecuencia de la baja tendencia a la formación de grumos $(5,7)$. De aquí el interés de promocionar el desarrollo de bebidas en polvo, donde se incorpore las harinas extrudidas de raíces y tubérculos.

Es importante resaltar que estos materiales amiláceos presentan en su composición química mayor contenido de amilopectina que de amilosa, lo cual contribuye a obtener suspensiones con mejores características espesantes, estabilizantes, consistencia del fluido viscoelástico, claridad de las pastas y estabilidad en un amplio intervalo de $\mathrm{pH}$ e incluso en agitación mecánica y en ciclos de congelación - descongelación, que son ideales para el desarrollo de este tipo de bebidas lácteas instantáneas (5 - 8).
Pacheco et al.(9) elaboraron mezclas en polvo para bebidas lácteas instantáneas a partir de harinas no convencionales como la harina pre-gelatinizada de plátano (Musa sp) y salvado de arroz (Oryza sativa L.), resultando una buena aceptación sensorial, atribuido según los autores a la relación equilibrada de la mezcla de estas harinas (25 y 12,8\% respectivamente), la cual fue calificada como un alimento con potencialidad nutritiva y calórica, por el contenido medianamente alto de proteínas $(7,25 \%)$, fibra dietaría $(12,8 \%)$ y de las proporciones de azúcares totales $(49,5 \%)$, mas que del almidón total $(23,7 \%)$, respectivamente y de la presencia del resto de los constituyentes, tales como la grasa $(1,56 \%)$, ceniza $(3,80 \%)$ y particularmente de almidón resistente $(2,05 \%)$.

Luiz (10) y Bou et al.(11) coincidieron en señalar que el uso de las harinas no convencionales de raíces y tubérculos tiende a crear un impacto favorable en los consumidores y de igual manera en la agroindustria, en esta última por representar una alternativa en el uso de otras harinas que poseen propiedades funcionales que se adaptan a sus requerimientos en la formulación de productos instantáneos y por tratarse de un producto que proviene de una producción autóctona de las regiones de la cordillera andina con bajos costos de producción y buenos rendimientos en harinas (18$30 \%$ ), al procesarlas por los métodos industriales de secado por convección.

Estas harinas extrudidas caracterizadas por tener una alta capacidad de re-hidratación, solubilidad y absorción de agua, son de interés para el desarrollo de productos instantáneos en polvo y muy convenientes por ser compatibles con otros ingredientes, como la leche entera, descremada o hidrocoloides, entre otros, que en conjunto favorecen el desarrollo de suspensiones viscosas con una fase continua y de alta estabilidad (12 - 14).

Otro aspecto novedoso en la formulación de las bebidas en polvo, es la adición de nutrientes, tales como vitaminas, minerales, aminoácidos, proteínas u otros, los que se han generalizado en la elaboración de alimentos especiales, por ser atractivos para los consumidores que continuamente buscan productos que provean beneficios a la salud. En este sentido, se citan los productos formulados con ácido fólico, recomendados para la mujer embarazada. El ácido fólico $(\mathrm{C} 19 \mathrm{H} 19 \mathrm{~N} 7 \mathrm{O} 6)$ corresponde a un nutriente clasificado entre las vitaminas del complejo B, que cumple numerosas funciones en el organismo en conjunto con la vitamina B12 y el aminoácido metionina, tanto para la prevención de anemia como en los neurotransmisores en el cerebro y en la síntesis del ADN incluso se sugiere para contrarrestar los daños en el desarrollo del tubo neural del niño durante 
la gestación (15 - 17). Dadas las premisas expuestas, el objetivo de este estudio fue formular y elaborar una bebida láctea instantánea con harina extrudida de arracacha (Arracacia xanthorrhiza) con la adición de ácido fólico y de un saborizante como la vainilla (Vanilla planifolia) para hacer atractivo el sabor de este producto ante el consumidor en general.

\section{MATERIALES Y MÉTODOS}

Elaboración de las bebidas: Para las pruebas preliminares de las formulación de una bebida tipo merengada, se tomó como referencia las sugerencias de la norma Covenin ${ }^{\circ} 2125$ (3) de mezclas deshidratadas para preparar bebidas instantáneas y las experiencias de Pacheco et al. (9) en la evaluación nutricional y sensorial de mezclas de polvos para bebidas instantáneas. Para la dosis a adicionar de ácido fólico a la mezcla en estudio se consideró las experiencias de Villaseñor y García (18) en harinas, como las de Barclay (19) en bebidas lácteas, quienes sugieren agregado de ácido fólico en polvo en $2 \mathrm{mg} / \mathrm{kg}$ (figura 1). De las pruebas preliminares resultaron cuatro formulaciones con la incorporación de la harina de arracacha de 25 a $33 \%$ (tabla 1), definiendo estas relaciones un producto del tipo merengada, donde los ingredientes (leche, azúcar, saborizante a base de vainillina, ácido fólico y harina de arracacha) permitieron desarrollar una bebida lista para consumir una vez reconstituida con agua.

Evaluación sensorial: Se realizó una evaluación sensorial afectiva de comparación pareada por prefe-

\section{FIGURA 1}

\section{Esquema de elaboración para la mezcla en polvo de la bebida láctea instantánea con base a harina extruida de arracacha y adición de ácido fólico.}

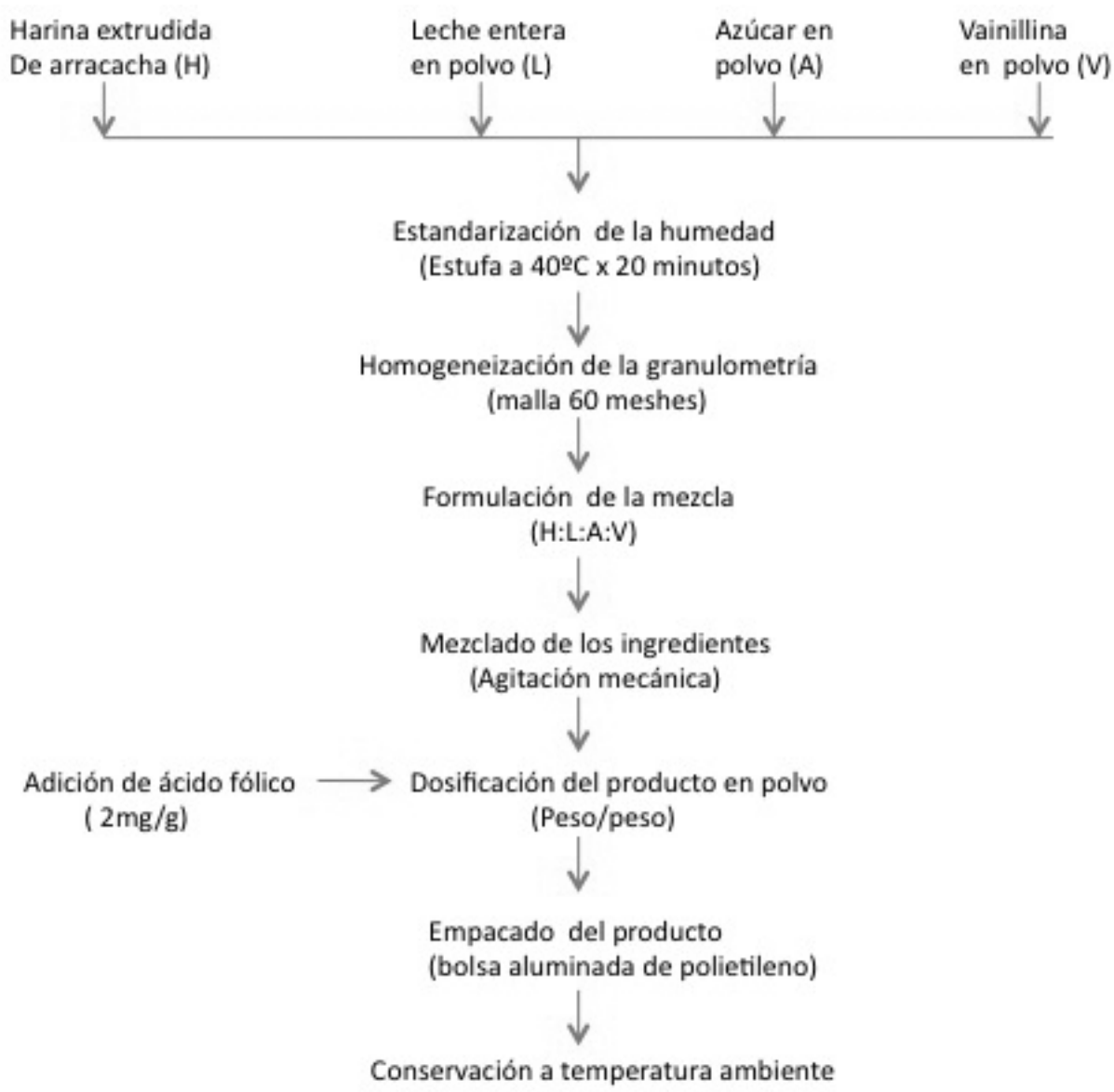


rencia con un panel de 50 jueces no entrenados. Los datos obtenidos se analizaron por mínimas respuestas concordantes (MRC) para un nivel de confianza del 95 $\%$ por la vía no paramétrica, para la selección de la fórmula de mayor preferencia. Esta muestra fue sometida a la determinación de las características físico-químicas, funcionales, análisis de la composición química, prueba de digestibilidad in vitro del almidón y estabilidad de sus características físico-químicas y funcionales durante el almacenamiento por tres meses a temperatura ambiente (20).

Características físico-químicas: Las determinaciones correspondieron al $\mathrm{pH}$ por el método $\mathrm{n}^{\circ} 1315-79$ (21) contenido de humedad siguiendo el método $\mathrm{n}^{\circ} 925.09$ (22), actividad del agua utilizando el equipo psicrométrico Equoi Aqualab Decagon cx-2-, color aplicando el método de Hunter Lab, perfil de viscosidad aparente de la bebida instantánea reconstituida a la temperatura de $25^{\circ} \mathrm{C}$ y diferentes tiempos de agitación (2,4,6,8,10 minutos) usando el viscosímetro Brookfield (Modelo LVF, serial 63127, Brookfield Laboratories Inc., Stoughton, MA, USA) con la aguja $\mathrm{n}^{\circ} 4$ a una velocidad de la fuerza de corte constante de 30 r.p.m, los datos de esta medida se reportaron en unidades de centipoises (cps) (9). Todas las determinaciones se hicieron por triplicado.

Composición química: Se determinó siguiendo las recomendaciones de la AOAC (22) el contenido de proteína (método $\mathrm{n}^{\circ} 979.09$ ), ceniza total (método $\mathrm{n}^{\circ}$ 923.03), grasa (método $n^{\circ} 920.39$ ), fibra dietética usando el método enzimático-gravimetrico ( $\mathrm{n}^{\circ}$ 985.29), azúcares totales y reductores ( método $\mathrm{n}^{\circ}$ 925.36) y el contenido de minerales, como el calcio por el método de espectrofotometría de emisión de luz, utilizando un fotómetro, marca Eppendorf , hierro (método ${ }^{\circ}$ 963.09) y fósforo (método $\mathrm{n}^{\circ}$ 965.17), mientras el almidón disponible se hizo por el método multienzimático de Holm et al.(23) y el almidón resistente utilizando el procedimiento de Goñi et al. (24) Todos los análisis se hicieron por triplicado y los valores se expresaron en porcentaje (\%). El valor energético o calórico, se calculó considerando los coeficientes de $4 \mathrm{kcal} / \mathrm{g}$ para carbohidratos y proteínas, $2 \mathrm{kcal} / \mathrm{g}$ para la fibra dietética y $9 \mathrm{kcal} / \mathrm{g}$ para los lípidos, expresando los resultados en $\mathrm{kcal} / 100 \mathrm{~g} \mathrm{(25).}$

Digestibilidad in vitro del almidón: Esta característica se determinó por la hidrólisis enzimática in vitro del almidón por el método de Holm et al.(26).

Características funcionales: Estas correspondieron a la determinación de la capacidad de absorción en agua del almidón por el método de Wang y Kinsella (27), solubilidad del almidón en agua fría según Eastman y Moore (28) y el poder de hinchamiento del almidón en agua fría siguiendo el método descrito por Ruales et al.(29). Las pruebas se realizaron por triplicado y los resultados se expresaron en porcentaje (\%).

Estabilidad durante el almacenamiento: La evaluación de la estabilidad en anaquel del producto empacado en bolsas aluminada de polietileno, se realizó a temperatura ambiente $\left(28+2^{\circ} \mathrm{C}\right.$ y $70 \%$ humedad rela-

\section{TABLA 1}

Formulaciones de las mezclas en polvo de la bebida láctea instantánea.

\begin{tabular}{lcccc} 
& Formulaciones (F) / Proporción (\%) & & F4 \\
\hline Ingredientes & F1 & F2 & F3 & F \\
\hline Harina extrudida de arracacha & 30,30 & 25,89 & 30,30 & 33,30 \\
Leche entera en polvo & 42,32 & 44,35 & ----- & ------ \\
Leche descremada en polvo & ----- & 42,32 & 41,62 \\
Azúcar en polvo & 27,22 & ----- & 24,92 \\
Vainillina en polvo & 0,16 & 0,16 & 27,22 & 0,12 \\
Acido fólico en polvo & 0,0002 & 0,0002 & 0,16 & 20000 \\
Viscosidad (cp) & 2000 & 1500 & 1500 & \\
\hline $\begin{array}{l}\text { F1: Harina extrudida de arracacha del morfotipo blanco } \\
\text { F3: Harina extrudida de arracacha del morfotipo amarillo }\end{array}$ & & & \\
F2: Harina extrudida de arracacha del morfotipo blanco & & \\
F4: Harina extrudida de arracacha del morfotipo amarillo & & \\
Viscosidad medida con la aguja ${ }^{\circ}$ 4 y con una fuerza de corte de 30 r.p.m, en el viscosímetro de Brookfield \\
(Modelo LVF, serial 63127, Brookfield Laboratories Inc., Stoughton, MA, USA).
\end{tabular}


tiva) por un periodo de tres meses, analizando cada cinco días el contenido de humedad, actividad del agua, color, $\mathrm{pH}$, viscosidad aparente, capacidad de absorción de agua, poder de hinchamiento y solubilidad de la mezcla en polvo en agua fría. Las pruebas se realizaron siguiendo los métodos antes descritos y por cuadriplicado.

Análisis estadístico: Los datos de las distintas evaluaciones se analizaron mediante una prueba estadística descriptiva a un nivel de significancia del $5 \%$, siguiendo un diseño completamente aleatorizado. Estos análisis se hicieron por el programa computarizado de Statistix, usando el soporte técnico de Windows 2000 (30).

\section{RESULTADOS Y DISCUSIÓN}

\section{Evaluación sensorial}

El resultado del análisis sensorial por el método de las mínimas respuestas concordantes (MRC) sobre la base de la distribución binomial de dos colas para un nivel de confianza del $95 \%$ (MRC:33), determinó que la mezcla en polvo de mayor preferencia con relación a los atributos de color, sabor, olor y viscosidad, fue la formulada con $30,30 \%$ de harina extrudida de arracacha del morfotipo blanco, 42,32 \% leche entera, 27,22 \% azúcar, $0,16 \%$ vainillina y $0,0002 \%$ ácido fólico $(\mathrm{F} 1)$ (tabla 2). Esta respuesta indicó la aceptación de las características organolépticas que promueve la harina de arracacha como ingrediente en el desarrollo de esta bebida tipo merengada, además de su posible grado de preferencia en otros tipos de mezclas en polvo para bebidas lácteas instantáneas, donde se incorpore en la misma proporción.

\section{Características físicoquímicas}

En la tabla 3 se presentan las características físicoquímicas de la bebida seleccionada de mayor preferencia, la cual se caracterizó por tener un contenido de humedad de 3,31 $\pm 0,02 \%$, que se encontró dentro del limite máximo del $5 \%$ especificado para mezclas de bebidas en polvo instantáneas indicado en la norma Covenin $\mathrm{n}^{\circ} 2125$ (3). Adicionalmente, este resultado tiende al intervalo promedio reportado por Pacheco et al.(9) de 3,10 a 3,24\% en bebidas a base de harinas no convencionales de plátano verde, aunque fue menor al rango de humedad de 6,80 a 7,00\%, determinado en una bebida láctea con la incorporación de la harina extrudida de ñame (Dioscorea alata) expresado por Pacheco et al.(7), y por el señalado de hasta $3,5 \%$ en una bebida láctea con base de cereales fortificada con vitaminas y minerales, referido por Vera et al. (31). El bajo contenido de humedad se consideró ideal para productos en polvo por mantener la alta capacidad de re-hidratación e higroscopicidad de la mezcla, tal como lo refieren los autores antes mencionados; a pesar de que desde el punto de vista operativo, está condición pueda causar problemas durante el mezclado de los ingredientes y mantener la adecuada dispersión del mismo para el empacado, como consecuencia de la facilidad que tiene el material de absorber agua del medio ambiente y rápidamente apelmazarse como lo explica Marín et al.(32). Este autor menciona que para asegurar una adecuada rehidratación y rápida solubilidad de la mezcla rápida en forma homogénea, se recomienda la hidratación en exceso de agua usando mezcladores a una alta veloci-

\section{TABLA 2}

Evaluación sensorial de las mezclas en polvo de la bebida láctea instantánea.

Formulaciones (F) / Proporción (\%)

Atributos sensoriales

Color

Olor

Sabor

Viscosidad
F1

165,0 a

157,5 a

190,0 a

179,0 a
F2

$156,0 \mathrm{c}$

$148,5 \mathrm{~b}$

$142,0 \mathrm{~d}$

$169,5 \mathrm{c}$
F3

$125,5 \mathrm{e}$

137, a5 c

$90,5 \mathrm{e}$

$110,0 \mathrm{e}$
$138,5 \mathrm{~d}$

F4

$148,5 \mathrm{~b}$

$182,5 \mathrm{~b}$

$173,0 \mathrm{~b}$

HAMB: harina extrudida de arracacha del morfotipo blanco, HAMA: Harina extrudida de arracaha del morfotipo amarillo,

LE: Leche entera, LD: Leche descremada. Az: azúcar, Va: Vainillina, AF: Acido fólico

F1: 30,30 \% HAMB, 42,32 \% LE, 27,22 \% Az, 0,16\% Va, 0,0002\% AF

F2: $25,89 \%$ HAMA, 44,35 \% LE, $29,56 \%$ Az 0,20\% va, 0,0002\% AF

F3: $30,30 \%$ HAMB, $42,32 \%$ LD, $27,22 \%$ Az, $0,16 \%$ Va, $0,0002 \%$ AF

F4: $33,30 \%$ HAMA, $24,96 \%$ LD, $27,22 \%$ Az, $0,16 \%$ Va, $0,0002 \%$ AF

Letras iguales en una misma fila indica que no existe diferencias estadísticamente significativas entre las muestras ( $\mathrm{p}>0,05)$. 
dad. Otra característica de importancia asociada al bajo contenido de humedad en este producto, fue la actividad de agua $\left(0,36 \pm 0,01 \mathrm{a}_{\mathrm{w}}\right)$, la cual resultó menor al compararla con otras mezclas de alimentos de preparación instantánea (040-0,48 $\mathrm{a}_{\mathrm{w}}$ ) reportados por Sáenz et al.(33) y Pacheco et al.(9). Esta baja actividad de agua tiende a ser ideal en productos secos, ya que valores menores a 0,6 aw, son indicativos de una condición que inhibe toda la actividad microbiana en la mezcla en polvo, y favorece la estabilidad de las propiedades físico-químicas, funcionales y hasta organolépticas. De manera que este valor de $\mathrm{a}_{\mathrm{w}}$, se consideró adecuado para este tipo de bebida láctea en polvo.

Con relación al color está muestra se caracterizó por presentar alta luminosidad indicativa de una mezcla con tendencia al blanco ( $\mathrm{L}: 85,90 \pm 0,07$ ), influenciada por una tonalidad con predominio del color amarillo, dado por el valor promedio del croma $b(15,92 \pm 0,04)$, con un matiz ligeramente oscuro atribuido al croma a $(-0,78 \pm 0,02)$, estableciendo una coloración similar al de una mezcla comercial en polvo de leche y cereales con sabor a vainilla. Se señala que esta medida resultó de aceptación y agrado por el panel no entrenado, durante la evaluación sensorial antes mencionada.

En referencia al $\mathrm{pH}$ se encontró un valor promedio de 6,66 $\pm 0,04$, que correspondió al ideal para productos de bebidas lácteas no ácida, similar a la indicada en la bebida láctea formulada con la incorporación de la harina extrudida de ñame (pH: 6,48-6,60) por Pacheco et al. (7) y a la especificada para las bebidas lácteas con base a cereales y sabor a vainilla $(\mathrm{pH}: 6,2-6,8)$ reportada por Vera et al. (31), avalando que la incorporación de la harina extrudida de arracacha, permite desarrollar esta característica de importancia en la palatabilidad del producto y que representó la aceptación de los jueces durante la evaluación sensorial de este producto.

$\mathrm{Al}$ analizar el perfil de viscosidad a una velocidad de corte constante (30 rpm) de la muestra reconstituida, se observó una disminución en función al tiempo del valor inicial de $2000 \pm 10$ cps a $1600 \pm 12$ cps en el transcurso de ocho (8) minutos, similar al comportamiento que caracteriza a los fluidos no newtoniano. Esta variación de la viscosidad en función del tiempo de agitación mecánica, se atribuyó a los cambios estructurales dados por la fuerza constante de corte sobre las moléculas de los distintos ingredientes de la mezcla, conllevando a que existiera una modificación en el equilibrio de estas a nivel del tipo de unión, fuerzas de atracción y repulsión, tal como lo observó Rodríguez et al. (13) en suspensiones de harina de arracacha. Sin embargo, este cambio de la viscosidad al parecer no afectó que la suspensión mantuviera una baja tendencia a la formación de grumos, aunque esta respuesta estuvo directamente relacionada con la modificación de gránulos de almidón al ser extrudidos.

\section{TABLA 3}

Características físico-químicas de la mezcla de mayor preferencia de la bebida láctea instantánea (F1)

\section{Característica física}

Humedad (\%)

Actividad de agua $\left(\mathrm{a}_{\mathrm{w}}\right)$

$\mathrm{pH}$

Color (método Hunter Lab)

L (Luminosidad (+) $100(-) 0$

a (+) color rojo (-) color verde

b (+)color amarillo (-) color azul

Viscosidad inicial (cps)

Viscosidad transcurridos ocho (8) minutos(cps)

Viscosidad final (cps)

\section{Muestra F1}

$$
\begin{aligned}
& 3,31+0,02 \\
& 0,36+0,01 \\
& 6,66+0,04
\end{aligned}
$$

$85,90+0,07$

$-0,78+0,02$

$15,92+0,04$

$2000+10,0$

$1600+12,0$

$1600+12,0$

Valor promedio \pm error estándar.

Muestra F1 (muestra de mayor preferencia): 30,30\% harina extrudida de arracacha del morfotipo blanco, $42,32 \%$ leche entera,

$27,22 \%$ azúcar, $0,16 \%$ vainillina y 0,0002 ácido fólico.

Viscosidad medida con la aguja $\mathrm{n}^{\circ} 4$ y con una fuerza de corte de 30 r.p.m, en el viscosímetro de Brookfield

(Modelo LVF, serial 63127, Brookfield Laboratories Inc., Stoughton, MA, USA). 
La viscosidad aparente promedio final, se consideró adecuada para este producto tipo merengada, por haberse evidenciado durante su preparación el desarrollo de un buen volumen asociado al alto poder de hinchamiento en frío y solubilidad de los gránulos de almidón aportados por la harina extrudida de arracacha, sin presencia de separación de fases. Estas características coincidieron con las exigencias de estabilidad de la suspensión para una bebida láctea instantánea, especificada por Vera et al. (31). Dada esta respuesta se consideró que la harina en estudio, resulta una propuesta adecuada para la formulación de este tipo de producto.

\section{Composición química}

La tabla 4, presenta la composición química de la mezcla seleccionada de mayor preferencia (F1), Se determinó que posee un contenido de proteína de 13,22 $\mathrm{g} / 100 \mathrm{~g}$, considerado alto y aportado por la leche entera en polvo $(27,40 \mathrm{~g} / 100 \mathrm{~g})$ y la harina de arracacha $(5,54$ $\mathrm{g} / 100 \mathrm{~g}$ ), ya que representaron los dos macro-ingrediente principales que suministraron esta proporción a la formulación.

Al comparar este resultado con las experiencias de Pacheco et al. (7) en formulaciones con harinas extrudidas de ñame, se comprueba que este aumento del contenido de proteína en las mezclas en polvo para preparar la bebida láctea, son dados por la incorporación de la leche, principalmente entera y por la harina de la tuberosa. Es importante desde el punto de vista nutricional formular alimentos donde se incluya como ingrediente el añadido de harinas de raíces y tubérculos. Por otra parte, Vera et al. (31) en una formulación para una bebida láctea instantánea, especificaron un alto contenido de proteína promedio de $19 \mathrm{~g} / 100 \mathrm{~g}$, el cual fue atribuido a la relación establecida entre la leche entera, las harinas de cereales, leguminosas, raíces y tubérculos, seleccionados para establecer la mezcla en polvo. Dados estos resultados y considerando que la ingesta diaria promedio de los adolescentes y adultos se encuentra en el intervalo de 0,8 a $1,0 \mathrm{~g} / \mathrm{kg}$ peso/día y de los niños entre 1 a $1,2 \mathrm{~g} / \mathrm{kg}$ peso/ día, la muestra en estudio F1, puede llegar a representar un buen aporte a esta ingesta por cada porción de 100 $\mathrm{g}$ de este alimento. Al analizar el contenido de grasa, se encontró que la muestra F1 tiene un promedio de 10,94 $\mathrm{g} / 100 \mathrm{~g}$, aportado por la grasa de la leche entera $(27,00$ $\mathrm{g} / 100 \mathrm{~g}$ ), más que por la harina extrudida de arracacha, debido a que está tiene muy baja proporción de lípidos $(0,48+0,03)$. El valor obtenido fue similar al especificado para la bebida láctea $(10 \mathrm{~g} / 100 \mathrm{~g})$ reportada por Vera et al.(31) y por Pacheco et al.(7) preparada con base a la harina extrudida de ñame $(10,80 \mathrm{~g} / 100 \mathrm{~g})$. Este contenido de grasa en la mezcla F1, se consideró alto e importante por el aporte de energía que puede hacer llegar al individuo por el consumo diario de esta bebida. Sin embargo, aunque este contenido puede ser cuestionado para la salud, en términos generales de nutrición humana,

\section{TABLA 4}

\section{Composición química de la bebida láctea instantánea (F1).}

\begin{tabular}{|c|}
\hline $\begin{array}{l}\text { Composición química } \\
(\mathrm{g} / \mathbf{1 0 0 g})\end{array}$ \\
\hline
\end{tabular}

Proteína

Grasa

Ceniza

Almidón

Almidón resistente

Azúcares totales

Azúcares reductores

Fibra dietaría

Calorías (Kcal/100g)

Vitamina: ácido fólico $(\mu \mathrm{g} / 100 \mathrm{~g})$
$13,22+0,02$

$10,94+0,09$

$4,02+0,03$

$22,02+0,04$

$0,44+0,01$

$46,68+0,07$

$24,36+0,09$

$3,12+0,03$

$417,41+1,20$

$200,00+0,01$
$5,54+0,03$

$0,42+0,02$

$3,64+0,02$

$72,28+0,04$

$1,49+0,01$

$8,50+0,03$

$3,86+0,04$

$9,62+0,04$

$368,30+0,09$

Valor Promedio + error estándar.

Muestra F1 (muestra de mayor preferencia): 30,30\% harina extrudida de arracacha del morfotipo blanco,

$42,32 \%$ leche entera, $27,22 \%$ azúcar, $0,16 \%$ vainillina y 0,0002 ácido fólico. 
la misma cumplen una acción estructural de recubrir y proporcionar consistencia a los órganos, catalizadores de reacciones químicas, funciones hormonales (esteroides) y de transportadores, tal como lo explican Sáenz et al. (33) y Sangronis et al.(34).

Desde el punto de vista de estabilidad de la mezcla durante el almacenamiento, un alto contenido de grasa puede originar oxidación (rancidez oxidativa) y promover características de olor y sabor no deseables, pero al respecto, se consideró lo señalado por García et al. (35) quienes indican que la técnica de secado por atomización empleada para la obtención de la leche entera en polvo, reduce esta tendencia, sobre todo cuando el alimento se mantiene bajo condiciones adecuadas de conservación y empacado.

Con relación a otros componentes se determinó que la ceniza en la muestra en estudio F1, tuvo un contenido promedio de 4,02 g/100g, que refleja el posible aporte de algunos minerales provenientes de los macro-ingredientes empleados para esta formulación con base a la leche entera y la harina extrudida de arracacha.

Con respecto a la fracción de almidón, se cuantificó un 22,02 g/100g, que representó el carbohidrato básicamente proveniente de la fuente de la harina extrudida de arracacha, cuya presencia juega un papel importante en la mezcla por contribuir al desarrollo de las características funcionales y de viscosidad de la bebida e intervenir en las características sensoriales del producto. El alto contenido promedio de azúcares totales $(46,68$ $\mathrm{g} / 100 \mathrm{~g})$ y reductores $(24,36 \mathrm{~g} / 100 \mathrm{~g})$ en la mezcla cumplió una función relevante por potenciar el sabor dulce y contribuir a establecer la rápida rehidratación de los componentes de la mezcla.

La fibra dietaria tuvo un contenido de $3,12 \mathrm{~g} / 100 \mathrm{~g}$, aportado por la harina extrudida de arracacha, ya que está posee un alto contenido $(9,62 \mathrm{~g} / 100 \mathrm{~g})$. Estos com- ponentes junto a la proporción de proteína y grasas, determinaron un valor de equivalente energético de $417,41 \mathrm{kcal} / 100 \mathrm{~g}$. Es importante señalar que la presencia de fibra dietaría, como del almidón resistente $(0,44 \mathrm{~g} / 100 \mathrm{~g})$ que al parecer tiene un comportamiento similar a la fibra dietaría, son de interés para la salud del hombre, ya que al consumirse diariamente a través de la bebida en estudio F1, facilitaría la disminución en el tiempo del tránsito intestinal, eliminando los problemas de estreñimiento y regulando la absorción intestinal de los azúcares procedentes de los alimentos, (36), Abdul y Luan (37) señalan que estos carbohidratos complejos, tienden a originar una resistencia a la digestión que conlleva a una disminución de la absorción de glucosa a nivel intestinal. En referencia al contenido de minerales (tabla 5) se determinó que la harina de arracacha contribuyó a incrementar el aporte de algunos minerales que provee la leche entera, a valores de calcio $(25,52 \mathrm{mg} / 100 \mathrm{~g})$, hierro $(32,56 \mathrm{mg} / 100 \mathrm{~g})$, fósforo $(9,07 \mathrm{mg} / 100 \mathrm{~g})$ y potasio $(395,20 \mathrm{mg} / 100 \mathrm{~g})$, que pueden ser de importancia en la nutrición y fisiología humana por la activación enzimática e intervención del sistema nervioso, excitabilidad y contractibilidad muscular (38-41). Es importante señalar que la biodisponibilidad de estos nutrientes, depende del tipo de sales, y de la capacidad de absorber y usar el cuerpo humano, que oscila en un promedio de 30 a $10 \%$ en adultos saludables (42). Con respecto a la adición del ácido fólico, se estimó que el consumo de $200 \mathrm{~g}$ de este producto, provee una dosis de 400 a $340 \mathrm{mg}$ de ácido fólico, considerando las pérdidas por preparación del alimento. De manera que el contenido presente en la bebida láctea F1, puede resultar en un buen equivalente para mejorar las concentraciones hemáticas de esta vitamina e incluso tener un efecto favorable en la prevención de defectos del tubo neural, aunque no

\section{TABLA 5}

\section{Contenido de minerales de la bebida láctea instantánea (F1).}

\section{Contenido de minerales $(\mathrm{mg} / \mathbf{1 0 0 g})$}

Calcio

Hierro

Fósforo

Potasio

\section{Muestra F1}

$25,52 \pm 0,33$

$32,56 \pm 0,11$

$9,07 \pm 0,08$

$395,20 \pm 1,80$

Valor de la media + error estándar

Muestra F1 (muestra de mayor preferencia): 30,30\% harina extrudida de arracacha del morfotipo blanco, 42,32 \% leche entera,

$27,22 \%$ azúcar, $0,16 \%$ vainillina y 0,0002 ácido fólico. 
óptimo, de acuerdo a lo indicado por Muzzo (43), quién recomienda la dosis adicionada en el producto en estudio en la dieta diaria.

\section{Características funcionales}

En relación a la capacidad de absorción de agua, se encontró un valor promedio de 3,07 $\pm 0,04 \mathrm{~g}$ agua $/ \mathrm{g}$ almidón (tabla 6), considerado alto y característico para este tipo de producto que se formula con harinas de raíces y tubérculos, así como en formulaciones con altos contenidos de proteínas, grasas, almidón y fibra dietaría. Villarroel et al. (44) y Belén et al. (45), obtuvieron una respuesta similar en formulaciones con base al uso de harinas no convencionales. Esta muestra presentó una alta solubilidad en agua fría con valores promedios de $96,66 \%$ y un incremento en su volumen, dado al aumento del poder de hinchamiento $(3,17 \mathrm{~g}$ gel/g almidón), relacionado más que con el tamaño de la partícula, cantidad de agua y tiempo determinado para su rehidratación y reconstitución, a la modificación del gránulo de almidón durante la obtención de la harina extrudida (7-14). Este tipo de harina extrudida conlleva al desarrollo de mezclas con alta solubilidad, viscosidad y estabilidad por la ausencia de separación de fases. Se

\section{TABLA 6}

Características funcionales de la bebida láctea instantánea (F1).

\section{Características funcionales}

Capacidad de absorción de agua (g agua/g almidón)

Solubilidad (\%)

Poder de hinchamiento del almidón (g gel/g almidón)

\section{Muestra F1}

$3,07 \pm 0,04$

$96,66 \pm 0,21$

$3,17 \pm 0,04$

Valor de la media + error estándar

Muestra F1 (muestra de mayor preferencia): 30,30\% harina extrudida de arracacha del morfotipo blanco, $42,32 \%$ leche entera,

$27,22 \%$ azúcar, $0,16 \%$ vainillina y 0,0002 ácido fólico.

\section{FIGURA 2}

\section{Digestibilidad in vitro del almidón en la mezcla en polvo de la bebida láctea instantánea con base a harina extruida de arracacha y adición de ácido fólico.}

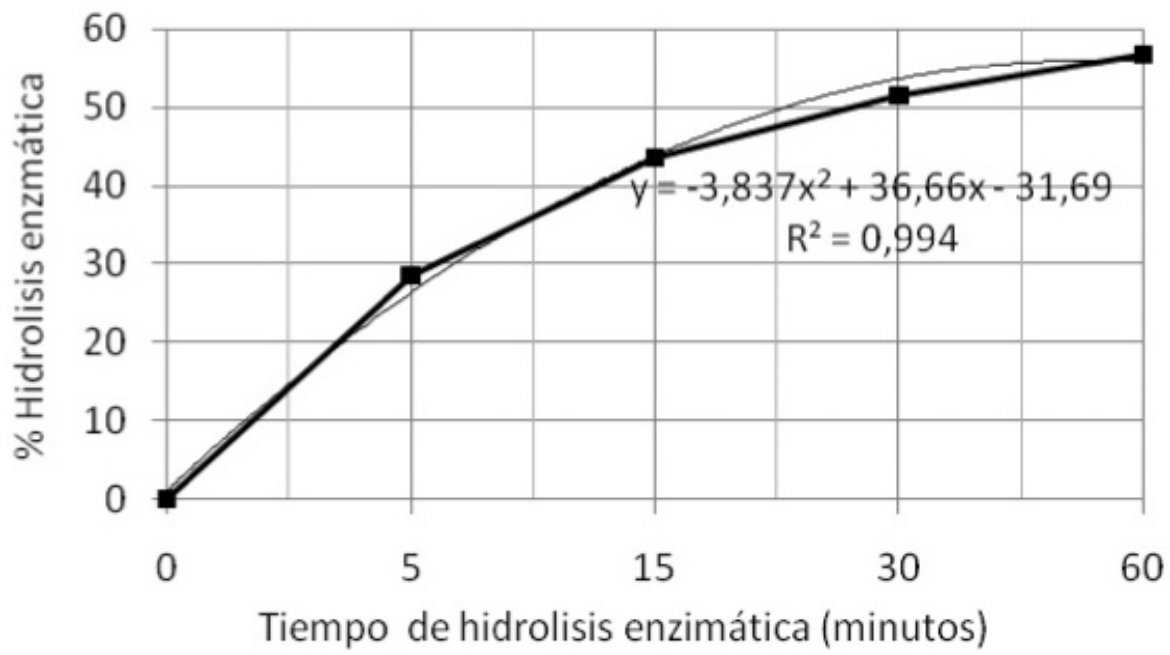


demostró que la harina extrudida de arracacha puede incorporarse a este tipo de formulación láctea, por permitir el desarrollo de una suspensión viscosa, que mantiene su estabilidad en una fase continúa, aún en presencia de los sólidos aportados por la leche entera en polvo y la azúcar, favoreciendo esta compatibilidad que pueda incorporarse una alta proporción de estos ingredientes, que no sólo contribuyen a elevar el valor nutricional y calórico, sino también a mejorar los atributos sensoriales en el producto reconstituido.

\section{Digestibilidad in vitro del almidón}

El análisis de hidrólisis enzimática, determinó que al cabo de los 15 minutos de la acción de la enzima $\alpha$-amilasa pancreática hubo una tasa de digestibilidad del almidón de 43,61 $\pm 0,12 \%$, con un incremento progresivo a los 60 minutos siguiendo una tendencia polinomial de segundo orden positiva $\left(\mathrm{R}^{2}: 0,994\right)$ hasta valores promedio de 56,80 $\pm 0,11 \%$, que indicaron una digestión intermedia de la mezcla al reconstituirla como bebida láctea instantánea (figura 2). Este grado de digestibilidad, se atribuyó a la presencia de fibra dietaria $(4,77 \pm 0,03 \mathrm{~g} / 100 \mathrm{~g})$ y almidón resistente $(0,44 \pm 0,01$ $\mathrm{g} / 100 \mathrm{~g}$ ), que de acuerdo a lo explicado por Zamora (46) en estudios de digestibilidad in vitro con una harina no convencional, son responsables de originar cierta resistencia a la actividad de la enzima para una mayor tasa de digestión o liberación de glucosa. Consideran que contenidos de fibra dietaría, almidón resistentes, una alta relación de proteína, así como los complejos que puedan existir incluso con los lípidos también influyen en esta respuesta.

\section{Estabilidad del producto durante el} almacenamiento a temperatura ambiente

Durante los tres meses de almacenamiento de la muestra de mayor preferencia (F1) a temperatura ambiente, se detectó que no hubo una variación del contenido de humedad (3,30 a 3,31\%), actividad de agua $\left(0,36 \mathrm{a}_{\mathrm{w}}\right), \mathrm{pH}(6,66)$ y color de la mezcla (tabla 7$)$. Se

\section{TABLA 7}

Humedad, actividad de agua, pH y color de la mezcla de mayor preferencia (F1) durante tres meses de almacenamiento.

\begin{tabular}{ccccccc}
$\begin{array}{c}\text { Tiempo } \\
(\mathbf{m e s e s})\end{array}$ & $\begin{array}{c}\text { Humedad } \\
(\boldsymbol{\%})\end{array}$ & $\begin{array}{c}\text { Actividad de } \\
\text { agua }\left(\mathbf{a}_{\mathbf{w}}\right)\end{array}$ & $\mathbf{p H}$ & \multicolumn{3}{c}{ Color (método Hunter Lab) } \\
\hline 1 & $3,30 \pm 0,01^{\mathrm{a}}$ & $0,36 \pm 0,01^{\mathrm{a}}$ & $6,66 \pm 0,01^{\mathrm{a}}$ & $85,90 \pm 0,03^{\mathrm{a}}$ & $-0,78 \pm 0,02^{\mathrm{a}}$ & $15,92 \pm 0,02^{\mathrm{a}}$ \\
2 & $3,31 \pm 0,01^{\mathrm{a}}$ & $0,36 \pm 0,01^{\mathrm{a}}$ & $6,66 \pm 0,01^{\mathrm{a}}$ & $85,89 \pm 0,02^{\mathrm{a}}$ & $-0,78 \pm 0,01^{\mathrm{a}}$ & $15,91 \pm 0,02^{\mathrm{a}}$ \\
3 & $3,31 \pm 0,02^{\mathrm{a}}$ & $0,36 \pm 0,01^{\mathrm{a}}$ & $6,66 \pm 0,01^{\mathrm{a}}$ & $85,88 \pm 0,02^{\mathrm{a}}$ & $-0,77 \pm 0,02^{\mathrm{a}}$ & $15,92 \pm 0,01^{\mathrm{a}}$
\end{tabular}

Letras iguales en una misma columna indican que no existen diferencias estadísticamente significativas de la variable en estudio con respecto a los meses de almacenamiento.

\section{TABLA 8}

Características funcionales y viscosidad de la mezcla de mayor preferencia (F1) durante tres meses de almacenamiento.

\begin{tabular}{ccccc}
$\begin{array}{c}\text { Tiempo } \\
\text { (meses) }\end{array}$ & $\begin{array}{c}\text { Capacidad de absorción de } \\
\text { agua (g agua/g almidón) }\end{array}$ & $\begin{array}{c}\text { Poder de hinchamiento } \\
(\mathbf{g} \text { gel/g almidón) }\end{array}$ & $\begin{array}{c}\text { Solubilidad } \\
(\%)\end{array}$ & $\begin{array}{c}\text { Viscosidad } \\
(\mathbf{c p s})\end{array}$ \\
\hline 1 & $3,06 \pm 0,01^{\mathrm{a}}$ & $3,18 \pm 0,01^{\mathrm{a}}$ & $96,66 \pm 0,01^{\mathrm{a}}$ & $1600 \pm 10^{\mathrm{a}}$ \\
2 & $3,07 \pm 0,01^{\mathrm{a}}$ & $3,18 \pm 0,01^{\mathrm{a}}$ & $96,65 \pm 0,01^{\mathrm{a}}$ & $1600 \pm 11^{\mathrm{a}}$ \\
3 & $3,06 \pm 0,01^{\mathrm{a}}$ & $3,17 \pm 0,01^{\mathrm{a}}$ & $96,66 \pm 0,01^{\mathrm{a}}$ & $1600 \pm 12^{\mathrm{a}}$
\end{tabular}

Letras iguales en una misma columna indican que no existen diferencias estadísticamente significativas de la variable en estudio con respecto a los meses de almacenamiento. 
observó que los valores de humedad, se encontraron en el intervalo de las especificaciones de calidad indicadas en la norma Covenin $\mathrm{n}^{\mathrm{o}} 2125$ (3). El comportamiento de las características de capacidad de absorción de agua (3,06 a 3,07 g agua/g almidón), poder de hinchamiento en agua fría (3,18 a 3,17 g gel/g almidón), solubilidad $(96,66$ a $96,65 \%)$ y viscosidad $(1600+10 \mathrm{cps})$ (tabla 8$)$, fue estable, en el rango de los valores promedio iníciales de esta muestra, sin mostrar diferencias estadísticamente significativas en función del tiempo, indicativo de que existe estabilidad del polvo de sus componentes en el empaque seleccionado a temperatura ambiente. Granito et al. (47) y Sandoval et al.(48) coinciden en señalar que las muestras en polvo con bajos contenidos de humedad y actividad de agua, como la muestra en estudio, favorecen que las características organolépticas y funcionales de las mezclas en polvo para preparaciones instantáneas se mantengan estables.

\section{CONCLUSIONES}

La formulación de una bebida láctea instantánea tipo merengada con base de harina extrudida de arracacha y adición de ácido fólico, resultó en un producto de aceptación sensorial, caracterizada por una composición química con alto contenido de proteína, grasa, almidón y fibra dietaría, que eleva el valor nutricional de este alimento, además de presentar un conjunto de características físicoquímicas y funcionales, que cumple con las especificaciones de la norma Covenin de mezclas en polvo para preparar bebidas instantáneas.

\section{RESUMEN}

Para diversificar el uso de la harina extrudida de arracacha, se planteó la elaboración de una bebida en polvo con la adición de ácido fólico. La metodología permitió determinar la composición química, características fisicoquímicas, funcionales, digestibilidad in vitro del almidón y estabilidad en anaquel. La formulación con $30,30 \%$ de harina, $42,32 \%$ de leche entera, $27,22 \%$ de azúcar y $0,16 \%$ de vainilla, fue la de mayor preferencia, caracterizada por una tasa de digestión intermedia y por desarrollar una suspensión viscosa tipo merengada, sin grumos, ni separación de fases, dada la alta absorción de agua, poder de hinchamiento y solubilidad de los gránulos del almidón. Se estimó que $200 \mathrm{~g}$ del producto provee entre 400 a $340 \mathrm{mg}$ de ácido fólico y un buen aporte de proteína, minerales y fibra dietaría. Durante tres meses de almacenamiento, se mantuvo la calidad inicial. En conclusión esta harina resultó una fuente de almidón alternativa para formular mezclas en polvo para bebidas lácteas instantáneas.

Palabras clave: Arracacia xanthorriza; arracacha; bebidas lácteas; digestibilidad in vitro del almidón; mezclas instantáneas.

Dirigir la correspondencia a:

Profesora

Auris García M

Laboratorio de Bioquímica de Alimentos

Instituto de Química y Tecnología

Facultad de Agronomía

Universidad Central de Venezuela

Maracay

Estado Aragua

Venezuela.

Código Postal 2105

E-mail: aurisgarcia@ hotmail.com

Agradecimientos: Los autores agradecen el financiamiento del CDCH-UCV, la ayuda de la técnica Gloria Betancourt y al Dr. Helis Hernández de la Fundación CIEPE.

\section{BIBLIOGRAFÍA}

1. Blanco A. Montero M. Fernández M. Composición química de productos alimenticios derivados de trigo y maíz elaborados en Costa Rica, ALAN 2000; 50(1): 91-6.

2. Salamanca G. Osorio M. Montoya L. Elaboración de una bebida funcional de alto valor biológico a base de borojo (Borojoa patinoi Cuatrec). Rev Chil Nutr. 2010; 37(1): 87-96.

3. Covenin (Comisión Venezolana de Normas Industriales). Mezclas deshidratadas para preparar bebidas instantáneas. n 2125:2001. 1era. Revisión. Publicación de Fondonorma, Categoría B. ICS. 67.260.20. Caracas, Venezuela. p 13, 2001.

4. Ibernorca. Reglamento para bebidas analcohólicas. Capitulo.4.2.3. Código RP.TCP.11.02.2004. NB.ISO.IEC.65, 2004; p 31.

5. Pacheco E. Evaluación nutricional de sopas deshidratadas a base de harina de plátano verde. Digestibilidad in vitro del almidón. Acta Cient Venez 2001; 52: 278-82.

6. Arcila N. Mendoza Y. Elaboration of an instant beverage amaranth seeds (Amaranthus cruentus) and its potential use in the human diet. Rev Fac Agron 2006; 23 (1):114-24.

7. Pacheco E. Techeira N. García A. Elaboración y evaluación de polvos para bebidas instantáneas a base de harina extrudida de ñame (Dioscorea alata). Rev Chil Nutr 2008; 35(4):452-9.

8. Shi X. Bimiller J. Effects of food gums on viscosities of starch suspensions during pasting.Carboh Poly- 
mers 2002; 50: 7-18.

9. Pacheco E. Pérez R. Schnell M. Evaluación nutricional y sensorial de polvos para bebidas a base de papaya, plátano verde y salvado de arroz. índice glucémico. Interciencia 2004; 29 (1):46-51.

10. Luiz E. Conceptos y tecnología para la elaboración y uso de las harinas compuestas. Inst Nutr Centro America y Panama 2006; 3: 11-3.

11. Bou L. Vizcarrondo C. Rincón A. Padilla F. Evaluación de harinas y almidones de mapuey (Dioscorea trifida), variedades blanco y morado. ALAN 2006; 56 (4):375-83.

12. Lamberti, M. Geiselmann A. Conde B. Escher F. Starch transformation and structure development in production and reconstitution of potato flakes. Food Sci Tech 2004; 37: 417-27.

13. Rodríguez E. Fernández. Quintero A. Alonso L. Ospina B. Reología de suspensiones preparadas con harina precocida de yuca. Rev Ing Desarrollo 2006; 19: 17-30.

14. Kadan R. Functional properties of extruded flours. J Food Sci. 2003; 68 (5): 1669- 72.

15. Ho J. Vyveganathan L. Jayaseelan S. Consumption of cereal flour in a Malaysian Population: Flour fortification to prevent neural tube defect may be feasible in a rice-eating country. Ecology Food Nutr 2006; 45(1):53-60.

16. Olivares A. Bernal M. Ros G. Calidad de los datos del contenido en ácido fólico en vegetales recogidos en varias tablas de composición de alimentos españolas, y nuevos datos sobre su contenido en folatos. Nutr Hospitalaria 2006; 21(1): 97-108.

17. Suárez M. Ácido Fólico: nutriente redescubierto. Acta Med Costarricense 2003; 45 (1): 5-9.

18. Villaseñor S. García P. Fortificación con ácido fólico. J American Nutr 2001; 2 (suppl): 129-134.

19. Barclay D. Fortificación múltiple de bebidas. Rev Centro Invest Nutr (Lausanne, Suiza) 2004: 20 (3S): $1-10$

20. Wittig E. El análisis sensorial en el desarrollo y evaluación de nuevos productos para regímenes especiales. Anales Univ Chil 2000; 6 (11):30-34.

21. Covenin (Comisión Venezolana de Normas Industriales). Determinación de $\mathrm{pH}$ (acidez ionica). 1era. Revisión. Publicación de Fondonorma, Categoría B. Caracas, Venezuela. p 11. 1979.

22. Association of Official Agricultural Chemists. Official Methods of Analysis of the AOAC. 15 th ed. Washington, D.C., The Association, p 980. 1990.

23. Holm J. Bjorck I. Drews A. Asp N. 1986 A rapid method for the analysis of starch. Starch/Starke 1986; 38:224-26.
24. Goñi I. García L. Mañas E. Saura A. Analysis of resistent starch: a method for foods and food products. Food Chem 1996; 56: 445-9.

25. Watt B. Merrill A. Composition of food. Handbook No 8. USDA Washington, D.C. p.355; 1963.

26. Holm J. Björck I. Asp N. Sjöberg L. Lundquist I. Starch availability in vitro and in vivo after flaking, steam-cooking and popping of wheat. J Cereal Sci. 1985; 3: 193-206.

27. Wang J. Kinsella J. Functional properties of novel proteins: alfalfa leaf protein. J Food Sci. 1976; 41: 286.

28. Eastman J. Moore C. Cold water soluble granular starch for gelled food composition. U.S. Patent 4465702. 6 p. 1984.

29. Ruales J. Valencia S. Fair B. Effect of processing on the physico-chemical characteristics of quinoa flour (Chenopodium quinoa W.). Starch/Stärke 45:13-19.

30. Montgomery, D. 1991. Diseño y análisis de experimentos. Editorial Iberoamericana. Caracas. p. 589; 1993.

31. Vera G. Alviña M. Araya H. Atalah E. El desarrollo de la formulación y de las especificaciones técnicas de la bebida láctea años dorados. Ministerio de Salud. Santiago de Chile-Gobierno de Chile. 49p. 2009.

32. Marin E. Lemus M. Flores M. La rehidratación de alimentos deshidratados. Rev Chil Nutr. 2006: 33 (3):527-38.

33. Saenz C. Sepulpeda E. Pak N. Uso de fibra dietética de nopal en la formulación de un polvo para flan. ALAN 2002; 52(4): 387-92.

34. Sangronis E. Teixeira P. Otero M. Guerra M. Hidalgo G. Manaca, batata y ñame: posibles sustitutos del trigo en alimentos para dos etnias del Amazonas venezolano. Arch Latinoam Nutr 2006; 56(1): 122-8.

35. García E. Gutierrez S. Nolasco H. Desarrollo de bebidas lácteas enriquecidas con ácidos grasos. Rev Tecn Láctea 2010; 36:5-10.

36. Jenkins D. Kendall C. Vuksan V. Viscous fibers, health claims and strategies to reduce cardiovascular disease risk. J Clin Nutr. 2000; 71: 401-2.

37. Abdul-Hamid A. Luan Y. Functional properties of dietary fibers from defatted rice bran. Food Chem. 2000; 68: 15-19.

38. Wolfgor R. Drago S. Rodríguez V. Pellegrino N. Valencia $M$. In vitro measurement of available iron in fortified foods. Food Res Int 2002; 35, 85-90.

39. Sedó P. Rodríguez S. Beneficios nutricionales del consumo de leche y productos lácteos para las personas adultas mayores. Campaña Panamericana del 
consumo de lácteos Costa Rica. p.32; 2008.

40. Molina E. Nuevas tendencias en el desarrollo de nuevos productos, los alimentos funcionales. Ind Alim 2000; 22 (4):9-15.

41. Hernández E. Guzma, V. Revisión: alimentos e ingredientes funcionales derivados de la leche. ALAN; 53(4): 333-47.

42. Gerstner G. Viabilidad de la fortificación con calcio de bebidas lácteas y de soya. Rev Mundo Lácteo y Cárnico 2009; 4: 20-3.
43. Muzzo S. Wheat flour fortification in latin american and the caribean region. Rev Chil Nutr. 2004; 31(3): 132-7.

44. Villarroel M. Uquiche E. Brito G. Optimización de formulaciones para productos dietéticos de pastelería. ALAN 2000; 50(1): 62-8.

45. Belén, C. Alemán D. Alvarez R. Moreno M. Evaluación de algunas propiedades funcionales y reológicas de harinas de coroba (Jessenia polycarpa Karst). Rev Fac Agron. 2004; 21 (2):161-71. 